\title{
Pharmaceutical Nanotechnology \\ Preparation of coated nanoparticles for a new mucosal vaccine delivery system
}

\author{
Olga Borges ${ }^{\mathrm{a}, \mathrm{b}, *}$, Gerrit Borchard ${ }^{\mathrm{a}, 1}$, J. Coos Verhoef ${ }^{\mathrm{a}}$, \\ Adriano de Sousa ${ }^{b}$, Hans E. Junginger ${ }^{a}$ \\ a Leiden/Amsterdam Center for Drug Research, Division of Pharmaceutical Technology, \\ P.O. Box 9502, 2300 RA Leiden, The Netherlands \\ b Center for Pharmaceutical Studies, Laboratory of Pharmaceutical Technology, Faculty of Pharmacy, \\ University of Coimbra, Rua do Norte, Coimbra 3000-295, Portugal
}

Received 2 December 2004; received in revised form 13 April 2005; accepted 23 April 2005

\begin{abstract}
It has been found that the adsorption of antigens onto chitosan particles is an easy and unique mild loading process suitable to be used with vaccines. In order to increase the stability of this particles and to prevent an immediate desorption in gastrointestinal fluids, a coating process with sodium alginate was developed. One of the challenges of this developing process was to keep the particles in the nanosized range in order to be taken up by M-cells of the Peyer's patches. The observed inversion of the particles' zeta potential values after coating suggested the presence of an alginate coating layer. These results were confirmed by FTIR and DSC techniques. Additionally, in vitro release studies showed that the presence of the alginate layer around the particles was able to prevent a burst release of loaded ovalbumin and to improve the stability of the nanoparticles in simulated intestinal fluid at $37{ }^{\circ} \mathrm{C}$. The optimisation of the coating process resulted in $35 \%(\mathrm{w} / \mathrm{w})$ for the loading capacity of the coated particles. SEM investigations confirmed a suitable size of the coated nanoparticles for the uptake by M-cells.
\end{abstract}

(C) 2005 Elsevier B.V. All rights reserved.

Keywords: Chitosan; Sodium alginate; Ovalbumin adsorption; Coated nanoparticles; Mucosal vaccination

\footnotetext{
* Corresponding author. Tel.: +351 239859927; fax: +351239827126.

E-mail address: olga@ci.uc.pt (O. Borges).

1 Present address: Enzon Pharmaceuticals, 20 Kingsbridge Road, Piscataway, NJ 08854, USA.
}

\section{Introduction}

In recent years, mucosal vaccination is being considered as a subject of great interest due to its advantages above the i.m. or s.c. application. The presence of specific antibodies in mucosal surfaces has long been recognized as the first barrier against pathogens entrance. The most effective way to induce 
mucosal immunity (i.e., secretory $\operatorname{IgA}$ ) is to administer a vaccine directly to the mucosal surface. Additionally, the existence of a common mucosal immune system allows successful targeting of vaccines to inductive compartments within mucosa-associated lymphoid tissues, inducing local humoral responses in lymphoid tissues at distant mucosal loci (Alpar et al., 1998). Both intranasal and oral routes have been used in several studies to achieve this goal. Particularly, the oral administration permits targeting of a suitable vaccineloaded delivery system to the ports of entry (so-called M-cells) of the largest inductive lymphoid tissue in the body, the intestine. The oral route is well accepted and easily allows the vaccination of large populations. However, the acidic environment of the stomach and the presence of enzymes make the oral delivery of vaccines a challenge where is difficult to achieve high and reproducible effects. In order to solve these difficulties, a considerable number of polymeric microparticulate systems are under investigation to deliver vaccines to the intestine while protecting them from adverse conditions that could affect their bioactivity (Singh and O'Hagan, 1998). Another important aspect is that these delivery systems could act as imunostimulants or adjuvants, increasing the immunogenicity of poor immune response antigens (Jabbal-Gill et al., 1999; Singh and O'Hagan, 1999).

Nevertheless, from a pharmaceutical perspective, it became evident that further advances in the formulation of delivery platforms needs to be introduced in order to increase both the stability of the antigens in the gastro-intestinal tract and the uptake of antigen-containing particles by the M-cells. One of the parameters that should be addressed is the size of the particles. It is well known that the size of the particles should be below $10 \mu \mathrm{m}$ in order to be taken up by M-cells of the Peyer's patches in the gut (Eldridge et al., 1991; Jani et al., 1992). Moreover, the preservation of antigen stability during encapsulation is also essential for the development of a successful controlled release vaccine delivery platform.

Chitosan microparticles as an oral and intranasal vaccine delivery system were already used in our group showing promising capabilities (Van der Lubben et al., 2001a,b, 2003; Bivas-Benita et al., 2003). In these studies, the vaccine was loaded by a mild and simple but effective adsorption method. By this method, deleterious preparation conditions, like elevated temperatures, high shear rates or the presence of organic solvents were avoided. This method has also been described by other groups that reported good adsorption capacities for different substances (Mi et al., 1999; Hejazi and Amiji, 2002). In the case that the chitosan particles are not very porous, the antigen will be preferentially adsorbed to the particle surface. This can cause stability problems because processes like desorption or the attack of the antigens by enzymes or acidic substances from the body fluids may occur. These obstacles may be overcome by coating those particles with an acid resistant polymer, like sodium alginate.

The two chosen polymers chitosan and sodium alginate, for this novel delivery platform are naturally occurring polysaccharides. They are polyelectrolyte polymers of opposite charges, biocompatible and biodegradable, and show a good safety profile. Furthermore they have been used as pharmaceutical excipients. Chitosan is the deacetylated form of chitin comprising copolymers of glucosamine and $\mathrm{N}$-acetyl glucosamine linked by $\beta-(1-4)$ linkages. The primary amino groups lead to special properties that make chitosan very interesting for pharmaceutical applications. Sodium alginate is also a hydrophilic polymer and comprises D-mannuronic (M) and L-guluronic acid (G) residues joined linearly by 1,4-glycosidic linkages (Johnson et al., 1997). The wide pharmaceutical applicability of alginates is, to a large extent, associated with their gel-forming capacity. Di- or polyvalent cations (calcium being the most widely studied example) can induce the gelation by cross-linking of the guluronic acid units (Rajaonarivony et al., 1993; Johnson et al., 1997). Sodium alginate has been used for preparing nanoparticles (Rajaonarivony et al., 1993; Gonzalez Ferreiro et al., 2002), microspheres (Wu et al., 1997; Fundueanu et al., 1999; Takka and Acarturk, 1999; Kulkarni et al., 2001; Chan et al., 2002; Coppi et al., 2002), microcapsules (Esquisabel et al., 2000) and beads (Kulkarni et al., 2001), for oral delivery. In particularly, the use of alginate microparticles as an antigen delivery system has been described in several publications and there are some indications that they are able to induce a mucosal and systemic immune response in a variety of animal species by both oral and intranasal administration (Cho et al., 1998; Bowersock et al., 1999; Rebelatto et al., 2001).

Over the last years, sodium alginate has also been used as a coating material for cells with some 
advantages. It seems that the coating acts as a barrier to microbial contamination, and thus improved survival prospects of the coated cells (Kampf et al., 2000). In another study, the coating is performed to protect donor mammalian cells against antibodies and cytotoxic cells of the host immune system, allowing the transplantation of cells in the absence of immunosuppression (de Vos et al., 2002).

This manuscript describes the development of a true nanocoating procedure, whereas other publications describing the entrapment of cells, liposomes (Machluf et al., 2000) or microspheres (Ramdas et al., 1999; Hejazi and Amiji, 2002) in an alginate gel matrix. This is, as far as we know, the first time that the construction of a nanosized alginate-coated chitosan delivery system is described with the particularly aspect that the antigen is adsorbed to the chitosan particles surface.

\section{Materials and methods}

\subsection{Materials}

Chitosan was purchased from Primex BioChemicals AS (ChitoClear ${ }^{\mathrm{TM}}$, Avaldsnes, Norway). According to the provider's specifications, the degree of deacetylation is $95 \%$ (titration method) and the viscosity is $8 \mathrm{cP}(1 \%$ solutions in $1 \%$ acetic acid). Low viscosity sodium alginate was kindly donated by ISP Technologies Inc. (MANUCOL LB ${ }^{\circledR}$, Surrey, UK). Ovalbumin (OVA; grade V; minimum 98\%) was purchased from Sigma Chemicals (St. Louis, USA) and all the others reagents used were of analytical grade. All solutions were prepared in Millipore water.

\subsection{Preparation of chitosan particles}

Chitosan particles were prepared by the precipitation/coacervation method described previously (Berthold et al., 1996). Shortly, chitosan was dissolved at a concentration of $0.25 \%(\mathrm{w} / \mathrm{v})$ in a solution with $2 \%$ (v/v) of acetic acid and $1 \%(\mathrm{w} / \mathrm{v})$ of Tween ${ }^{\circledR} 80$. The formation of the particles was achieved after the addition of $3.5 \mathrm{ml}$ of sodium sulfate solution $(10 \%, \mathrm{w} / \mathrm{v})$ to $200 \mathrm{ml}$ of the chitosan solution. The addition was made at a rate of $1 \mathrm{ml} / \mathrm{min}$ under mild agitation $(<50 \mathrm{rpm})$ and continuous sonication (vibracell sonicator; $600-\mathrm{W}$ model (output control "1"); Sonics \& Materials, Inc., Danbury, USA). Sonication was maintained for additional $15 \mathrm{~min}$ and the agitation for $60 \mathrm{~min}$ at room temperature (RT). The suspension was centrifuged for $30 \mathrm{~min}$ at $3500 \mathrm{rpm}(2800 \times \mathrm{g})$ and the supernatant was discarded. The particles were re-suspended twice in Millipore water, centrifuged again for $30 \mathrm{~min}$ and the supernatants were discarded. The particles were frozen in liquid nitrogen and freeze-dried overnight using a Christ freeze-dryer (Osterode am Harz, Germany). The dry powder was kept frozen until further use.

\subsection{Loading of the particles with ovalbumin}

The first step of the loading procedure was the suspension of the freeze-dried particles in a phosphate buffer (pH 7.4) placed in an ultrasound bath for $30 \mathrm{~min}$ in order to disaggregate the particles. The loading was done by incubating a solution of ovalbumin with chitosan particles under mild agitation at room temperature. The various concentrations used are presented in Table 1.

The loading efficacy and the loading capacity of the uncoated particles were calculated by an indirect way, quantifying the protein that remained in solution. After incubation, an aliquot of the particle suspension was centrifuged at $14,000 \mathrm{rpm}$ for $30 \mathrm{~min}$ and the protein in supernatant was quantified by BCA-protein assay (PIERCE, Rockford, USA) using a microplate reader with a $590 \mathrm{~nm}$ filter (Bio-Rad model 550, Veenendaal, The Netherlands). The absorbance reading value was corrected subtracting the average absorbance reading obtained in the BCA-protein assay from that one of the supernatants of unloaded nanoparticles prepared exactly in the same conditions. The corrected OD value was then used to calculate the concentration using the standard curve prepared at same time from individual ovoalbumin standards.

The drug loading capacity (LC) and loading efficacy (LE) were calculated from the following equations:

LC $(\%, w / w)=($ total amount of ovalbumin - nonbound ovalbumin)/weight of the particles $\times 100$ LE $(\%)=($ total amount of ovalbumin - non-bound ovalbumin)/total amount of ovalbumin $\times 100$ 


\subsection{Coating of the nanoparticles with alginate}

Various amounts of the ovalbumin-loaded particle suspension were added under agitation to various solutions of sodium alginate (Table 1). The suspension of the particles was maintained under agitation with a magnetic stirrer for $20 \mathrm{~min}$ at RT. The suspension was then centrifuged for $10 \mathrm{~min}$ at $1600 \mathrm{rpm}$ and the supernatant was discarded. To chemically cross-link the alginate at the particle's surface, the particles were re-suspended in $0.524 \mathrm{mM} \mathrm{CaCl} 2$ solution and kept under agitation for another $10 \mathrm{~min}$. For the characterization of the nanoparticles (Section 2.5) the optimised formulation batch was used as given in Table 1 (system D).

\subsubsection{Evaluation the desorption during the coating procedure}

During the incubation of the particles with sodium alginate, aliquots of the particles suspension were collected, centrifuged at $14,000 \mathrm{rpm}$ for $30 \mathrm{~min}$ and the protein in the supernatant was assayed with a BCAprotein assay as described in Section 2.3.

Statistical methods used in this section include descriptive statistics (arithmetic mean and standard deviation) and Student's $t$-test.

\subsection{Characterization of the nanoparticles}

\subsubsection{Morphology}

The morphology and surface appearance of the particles were examined by scanning electron microscopy (SEM). One drop of the particles suspension was placed on a gold-coated plate and maintained at least $12 \mathrm{~h}$ at room temperature in a desiccator for complete dryness of the sample. The dry samples were coated with a thin gold layer using Emitech K650X large sample coater (Emitech, Kent, UK) and observed with a Jeol JSM$6700 \mathrm{~F}$ field emission scanning electron microscope (JEOL BV, Schiphol-Rijk, The Netherlands).

\subsubsection{Size and zeta potential measurements}

The particle size and zeta potential were evaluated by a dynamic light scattering technique with a Zetasizer 3000HSA (Malvern Instruments, Bergen op Zoom, The Netherlands). Zeta potential determinations were based on electrophoretic mobility of the nanoparticles in diluted aqueous suspensions. These measurements 
were performed at least in triplicate with independent particle batches.

\subsubsection{FT/IR studies}

The coated particles were washed with Millipore water, centrifuged and the sediment was freeze-dried overnight (Labconco, Kansas City, USA). The coated and uncoated particles were kept in desiccator at room temperature until analysis. The IR spectra of the samples were recorded using a Fourier-transformed infrared spectrophotometer instrument FT/IR-420 Jasco (Jasco Inc., Tokyo, Japan) with attenuated total reflection (ATR).

\subsubsection{Differential scanning calorimetry (DSC)}

DSC scans were recorded using a differential scanning calorimeter (DSC-50, Shimadzu Co., Kyoto, Japan). Two to four milligrams of the dry particles were accurately weighed into aluminium pans without seals and heated from 25 to $350^{\circ} \mathrm{C}$ at a heating rate of $10^{\circ} \mathrm{C} / \mathrm{min}$ under a nitrogen flow of $20 \mathrm{ml} / \mathrm{min}$.

\subsection{In vitro release studies}

The ovalbumin release from the coated and uncoated particles was performed in simulated intestinal fluid (SIF) as described in USP XXIV. The nanoparticles suspensions were added (1:4) to individual tubes containing the release medium previously equilibrated at $37^{\circ} \mathrm{C}$ and placed in a shaker bath adjusted to $50 \mathrm{rpm}$. At appropriate time intervals, samples from each tube were filtered with a low protein-binding filter $\left(\right.$ MILLEX $^{\circledR} \mathrm{GV}-0.22 \mu \mathrm{m}$; durapore PVDF membrane; Millipore, Malsheim, France) followed by centrifugation for $20 \mathrm{~min}$ at $14,000 \mathrm{rpm}$ and the ovalbumin in supernatant assayed with a BCA-protein assay. Simultaneously, coated and uncoated blank nanoparticles suspensions were submitted to the same conditions and the filtered samples were used as blanks for correction of the BCA-protein assay as described in Section 2.3.

For the determination of the total protein content, $0.5 \mathrm{ml}$ of the suspensions of the coated and uncoated particles were incubated with $1.5 \mathrm{ml}$ of $0.085 \mathrm{~N}$ hydrochloric acid solution ( $\mathrm{pH} 1.2)$ in an ultrasound bath for $30 \mathrm{~min}$, followed by $3 \mathrm{~h}$ in a water bath at $37^{\circ} \mathrm{C}$. The samples were filtered and the protein was assayed by the BCA-protein assay. Suspensions of unloaded particles were analysed under the same conditions and were used as a blank for the correction of the OD value of the samples analysed with BCAprotein assay as described in Section 2.3. Additionally, the unbound ovalbumin in the particle's suspension was also determined in order to calculate the amount of the ovalbumin encapsulated in the beginning of the assay.

All experiments were performed at least in triplicate.

\subsection{SDS-polyacrylamide gel electrophoretic (PAGE) analysis of released ovalbumin}

Samples from the loading and coating particle suspensions were centrifuged at $14,000 \mathrm{rpm}$, the supernatants were collected and the concentration was adjusted in order to have the same theoretical concentration (i.e., assumption of $0 \%$ of loading efficiency) of the protein. Alginate-coated chitosan nanoparticles loaded with ovalbumin were diluted (1:5) in buffer phosphate $\mathrm{pH} 7.5$ at $37^{\circ} \mathrm{C}$ and incubated overnight at $50 \mathrm{rpm}$. An aliquot was collected and centrifuged. The ovoalbumin samples were then suspended in the loading buffer and heated for $5 \mathrm{~min}$ at $100^{\circ} \mathrm{C}$ just before the run.

The SDS-PAGE was performed with gels composed of $12 \%$ acrylamide, cast and run in Tris-glycine buffer. Gels were stained with $0.1 \%$ Coomassie brilliant blue in $10 \%$ of acetic acid in a solution of methanol:water $(1: 1)$.

\section{Results and discussion}

\subsection{Preparation of the vaccine delivery system}

The preparation of the delivery system contains three main steps, the manufacturing of the chitosan particles, their ovalbumin loading by adsorption and finally the coating with sodium alginate. The formation of the chitosan particles by a precipitation process with sodium sulphate has been described by several authors (Berthold et al., 1996; Roy et al., 1999; Van der Lubben et al., 2001b). They all describe an existing correlation between the necessary amount of sulphate ions, the molecular weight and deacetylation degree of the polymer. The loading of the cationic particles with the protein is a very mild process, which can be achieved by suspending the particles in a solution of the protein 


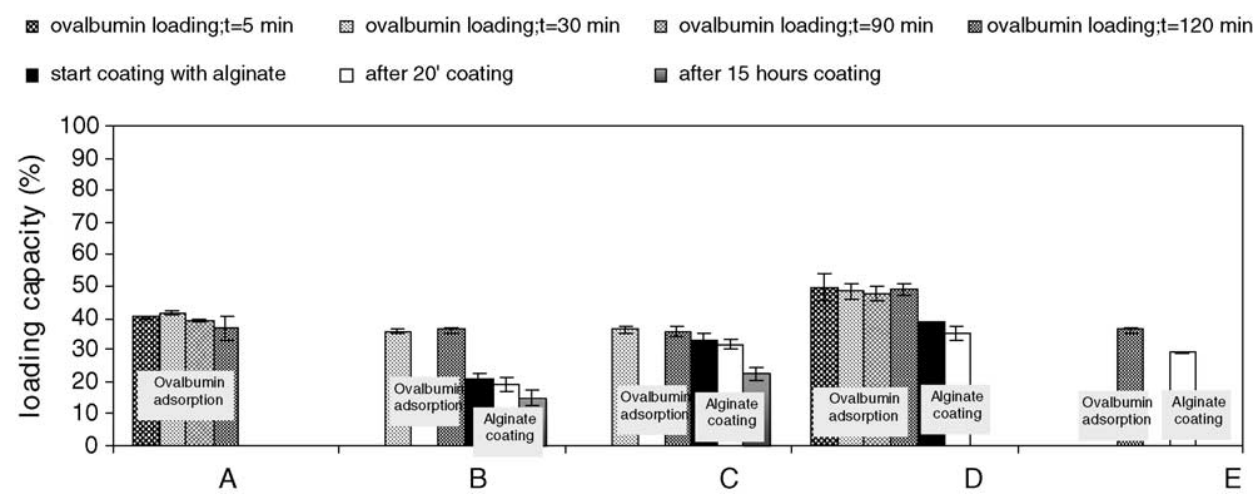

Fig. 1. Loading capacity of the particles during the different stages of the coated particles preparation. Results for the different systems (A-E).

in an appropriate buffer. The adsorption of the protein onto chitosan particles is mainly caused by ionic interaction of the chitosan amino groups with the carboxyl groups of protein substrate in a buffer phosphate solution of $\mathrm{pH} 7.4$ with high buffer capacity. According to previous work done in our laboratory (Van der Lubben et al., 2001b), the loading capacity of ovalbumin at a chitosan particles concentration of $1 \%(\mathrm{w} / \mathrm{v})$ is not substantially influenced in the range of $0.5-2 \%(\mathrm{w} / \mathrm{v})$ ovalbumin. Therefore, an ovalbumin concentration of $0.5 \%(\mathrm{w} / \mathrm{v})$ was used as loading solution resulting in a sufficient high loading capacity. For instance, for the system A, as shown in Figs. 1 and 2, the loading capacity and the loading efficacy were about 40 and $80 \%$, respectively. It was also found from the measurements of the unbound protein at different time points during the loading process that the adsorption equilibrium is rapidly reached. This means that the protein is bound to the surface of the particles and that the adsorption process of the ovalbumin to the particles surface occurs immediately after the addition of the protein solution to the particle suspension. Better loading capacity results $(p<0.00001)$ of the uncoated particles were found when the ratio particles/ovalbumin in the loading suspension was decreased from 2 (Table 1 systems A, B and E) to 1.6 (Table 1 system D) while the loading efficacy (uncoated particles) was not different $(p=0.15)$ between the systems.

The third step of the preparation was the coating of the chitosan nanoparticles with alginate solution.

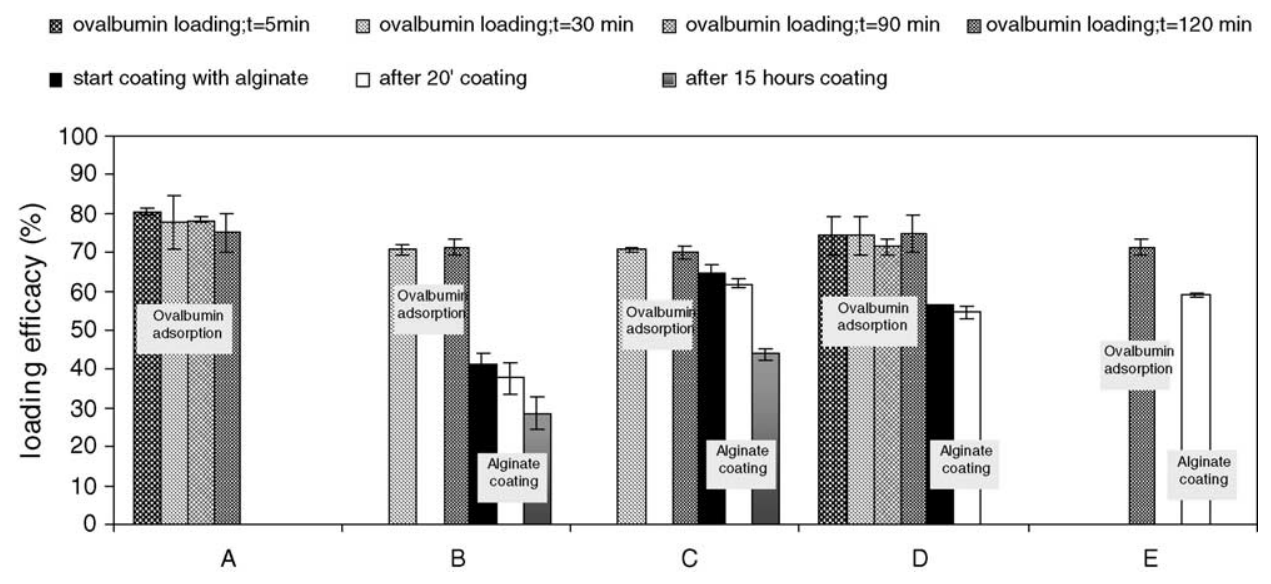

Fig. 2. Loading efficacy of the particles during the different stages of the alginate-coated particles preparation. Results for the different systems (A-E). 
To our knowledge this coating process has not been described in literature earlier. Consequently, the first part of this work is focused on the selection of the appropriate conditions and experimental methodology for the coating process with sodium alginate. For that purpose several systems with different ratios sodium alginate/chitosan particles have been investigated with the main objective to obtain a stable particle suspension. In the majority of the first trials an immediate flocculation was observed, particularly in systems with higher particles concentration. The formation of these agglomerates was easily observed because of the formation of a precipitate and a clear supernatant. Systems with a ratio alginate/particles $>2$ have shown to be stable. Thus, in the following optimization steps of the coating methodology always ratios higher than 2 were used.

Another important parameter studied was the desorption of ovalbumin from the particles during the coating process. The addition of the sodium alginate solution to the suspension of the loaded particles resulted in a new adsorption equilibrium characterized by the different concentration of protein and by the presence of alginate polymer that can compete with the interaction of the charges at the particle's surface. A significant decrease of the loading capacity of the coated particles was observed in all the systems $(p<0.05$ for systems B-E). Furthermore, we have observed that modifications in the $\mathrm{pH}$ of the coating medium can also modify the adsorption equilibrium of the protein. In our first experiments, the coating process was carried out at $\mathrm{pH}$ 5.5. This $\mathrm{pH}$ value seems to be the most favourable for the interaction between chitosan and alginate as the $\mathrm{p} K_{\mathrm{a}}$ of the chitosan is around 6.5 and the $\mathrm{p} K_{\mathrm{a}}$ of the sodium alginate is between 3.4 and 4.4. However, at this $\mathrm{pH}$ value, we have observed more than $60 \%$ of ovalbumin desorption (data not shown). For that reason a $\mathrm{pH} 7.4$ was adopted as it was observed to have the better compromised between loading capacity and efficiency of the coating process. The highest loading capacity for the alginate-coated nanoparticles was achieved with system D $(p=0.086$ when compared with system $\mathrm{B}$ and $p=0.032$ compared with system E). On the other hand, the comparison with system B $(p=0.001)$ and $\mathrm{E}(p=0.004)$ showed, that this option (system D) was achieved on the expenses of a slight decrease of the loading efficacy (Fig. 2).

\subsection{Characterization of the nanoparticles}

\subsubsection{Morphology, size and zeta potential measurements}

The precipitation of chitosan with sodium sulphate using ultrasounds for homogenisation led to the formation of particles in the nanoscale size (Table 2). One of the major currently described drawbacks of this technique is the high polydispersity of the obtained nanoparticles (Tang et al., 2003). We have observed nanoparticles with sizes ranging between 100 and $1000 \mathrm{~nm}$. The mean hydrodynamic diameter of the obtained particles after the precipitation process was $684 \mathrm{~nm}$ and the size increased after the freeze-drying process (Table 2). This is a direct consequence of particle aggregation during the drying process. To overcome particle aggregation the use of trehalose as a lyoprotectant was tried at concentrations of 3.3, 5 and $7 \%$ $(\mathrm{w} / \mathrm{v})$. A complete redispersibility of the freeze-dried nanoparticles in all trehalose concentrations and the maintenance of the particle size could be observed by light microscopy and dynamic light scattering technique. However, we optimised the duration of the freeze-drying process in order to avoid the use of cryoprotectants as they would interfere with the coating process.

SEM observations of the coated particles (Fig. 3b) indicated that the size range of the particles remained unchanged. This result indicates the feasibility of coating of ovalbumin-loaded chitosan nanoparticles with a thin layer of alginate. SEM images (Fig. 3a) showed some small particles $(<100 \mathrm{~nm})$ and a particle's agglomerate. However, it is not clear if this is a consequence of the freeze-dry process or if it is a consequence of the sample preparation (particle's concentration of the sample) for the SEM.

The stability of many colloidal systems is directly related to the magnitude of their zeta potential. In general, if the value of the particle zeta potential is large, the colloidal system will be stable. Conversely, if the particle zeta potential is relatively small, the colloid system will agglomerate. The surface charge of the particles is of substantial importance in all the production steps of these coated particles because the efficiency of the different steps is directly related to the establishment of electrostatic interactions. Chitosan particles showed a positive value of about $37 \mathrm{mV}$ (Table 3 ), which explains the stability of the particle suspensions in water and in 
Table 2

Size of the chitosan nanoparticles in different stages of the preparation process

\begin{tabular}{lll}
\hline & Cumulant $\mathrm{Z}$ average (nm), mean \pm S.D. & Polydispersity, mean \pm S.D. \\
\hline After the precipitation process & $643.2 \pm 171.7$ (16 batches) & $0.379 \pm 0.168(16$ batches $)$ \\
After lyophilization process & $955.6 \pm 161.0$ (3 batches) & $0.387 \pm 0.129$ (3 batches) \\
\hline
\end{tabular}

several buffer systems. After adsorption of ovalbumin, the particles are still positively charged with values higher than $30 \mathrm{mV}$. During the coating procedure an inversion of the surface charge of the particles to negative values was observed. This zeta potential inversion explains the difficulties that were found in preventing the formation of particles agglomerates during the coating process. After complete adsorption of the alginate to the particles, a surface charge of the particles of about $-35 \mathrm{mV}$ was found (Table 3 ). This zeta potential inversion is a strong indication of the presence of an alginate coating on the surface of the particles.
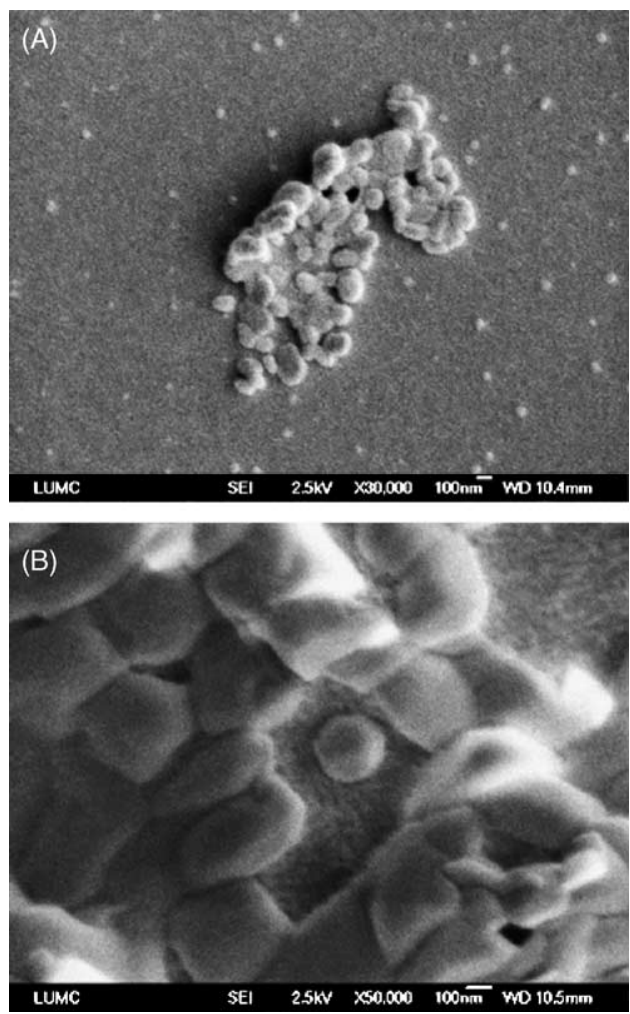

Fig. 3. Scanning electron micrographs of chitosan nanoparticles. (A) After freeze-dry and ressuspending in water. (B) After the coating procedure with sodium alginate.
Table 3

Zeta $(\zeta)$ potential of unloaded, ovalbumin-loaded chitosan nanoparticles and alginate-coated chitosan nanoparticles

\begin{tabular}{ll}
\hline & $\begin{array}{l}\text { Zeta potential }(\mathrm{mV}), \text { mean } \\
\text { average } \pm \text { S.D. }\end{array}$ \\
\hline $\begin{array}{l}\text { Empty chitosan } \\
\text { nanoparticles }\end{array}$ & $+37.0 \pm 3.6$ (seven batches) \\
$\begin{array}{l}\text { Ovalbumin-loaded } \\
\text { chitosan particles }\end{array}$ & $+41.3 \pm 6.4$ (five batches) \\
$\begin{array}{l}\text { Alginate-coated } \\
\text { chitosan particles }\end{array}$ & $-34.9 \pm 8.3$ (five batches) \\
\hline
\end{tabular}

\subsubsection{Differential scanning calorimetry}

As shown in Fig. 4, the DSC scans of the chitosan polymer exhibited an endothermic peak at about $66^{\circ} \mathrm{C}$ that has been attributed to the evaporation of absorbed water. The exothermic baseline deviation beginning around $250{ }^{\circ} \mathrm{C}$ indicates the onset of chitosan degradation (Khalid et al., 2002). The analysis of the DSC curves for chitosan particles showed two additional endothermic peaks at about 237 and $275^{\circ} \mathrm{C}$. The peak at $237^{\circ} \mathrm{C}$ is probably related to the breakdown of weak unspecific electrostatic interactions. The second peak is probably related to the cleavage of the electrostatic interactions between the polymer and the sulphate ions.

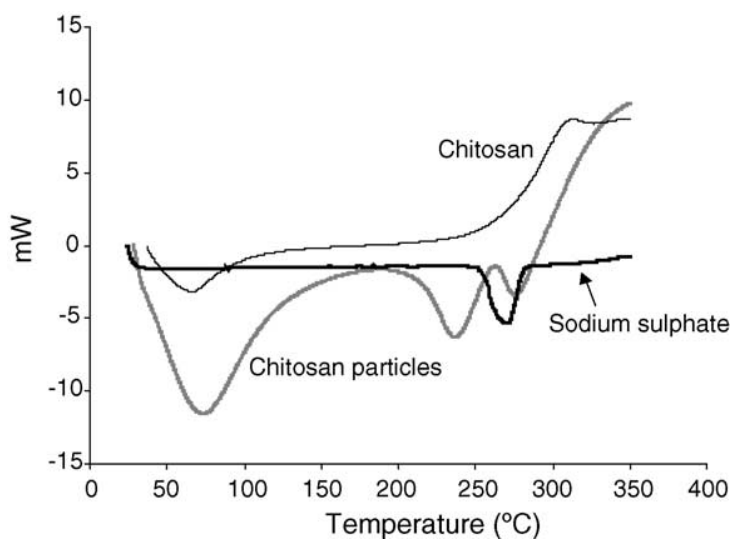

Fig. 4. Differential scanning calorimetry curves of chitosan, unloaded chitosan particles and sodium sulphate. 


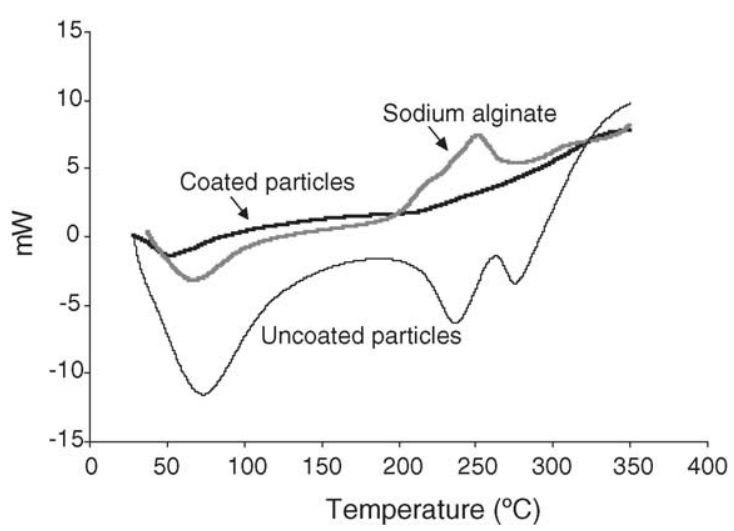

Fig. 5. Differential scanning calorimetry curves of sodium alginate, uncoated chitosan particles and alginate-coated particles. The DSC investigation was conducted using the unloaded particles.

After the coating process with sodium alginate, the particles exhibited a completely different behaviour as shown in Fig. 5, which means that an alteration occurred in the composition of the particles. In the DSC scans, the two endothermic peaks disappeared and no endothermic peak was found in the temperature range studied. In fact, a gradual appearance of an exothermic behaviour was detected starting around $200^{\circ} \mathrm{C}$ that coincides with the exothermic behaviour of the sodium alginate as referred to in several publications (e.g.,
Gonzalez-Rodriguez et al., 2002) as the decomposition of the polymer. These observations strongly support the presence of alginate molecules linked to the surface of the chitosan particles: when the temperature of the sample reaches values around $200{ }^{\circ} \mathrm{C}$, the two phenomena, the exothermic contribution from the alginate and the endothermic contribution from the chitosan particles determine the shape of the curve.

\subsubsection{FTIR characterization}

The FTIR spectra of chitosan and the chitosan particles, sodium alginate, and the coated particles are shown in Figs. 6 and 7, respectively.

In the chitosan spectra the strong and broad peaks in the $3400-3200 \mathrm{~cm}^{-1}$ ranges correspond to combined peaks of $\mathrm{O}-\mathrm{H}$ stretching and intermolecular hydrogen bonding. The $\mathrm{N}-\mathrm{H}$ stretching from primary amines are overlapped in the same region.

The $\mathrm{C}=\mathrm{O}$ stretching (amide) peak near $1633 \mathrm{~cm}^{-1}$ and $\mathrm{N}-\mathrm{H}$ bending (amide and amine) peak near $1542 \mathrm{~cm}^{-1}$ was observed as well. The intense peak at $1414 \mathrm{~cm}^{-1}$ belongs to the $\mathrm{N}-\mathrm{H}$ stretching of the amide and ether bonds give the peaks in the fingerprint region of the spectra, where the symmetric stretch of $\mathrm{C}-\mathrm{O}-\mathrm{C}$ is found around wave numbers of $1065-1027 \mathrm{~cm}^{-1}$. In the chitosan particles the peak of $1558 \mathrm{~cm}^{-1}$ is shifted to $1535 \mathrm{~cm}^{-1}$ and the relative intensity of this peak is

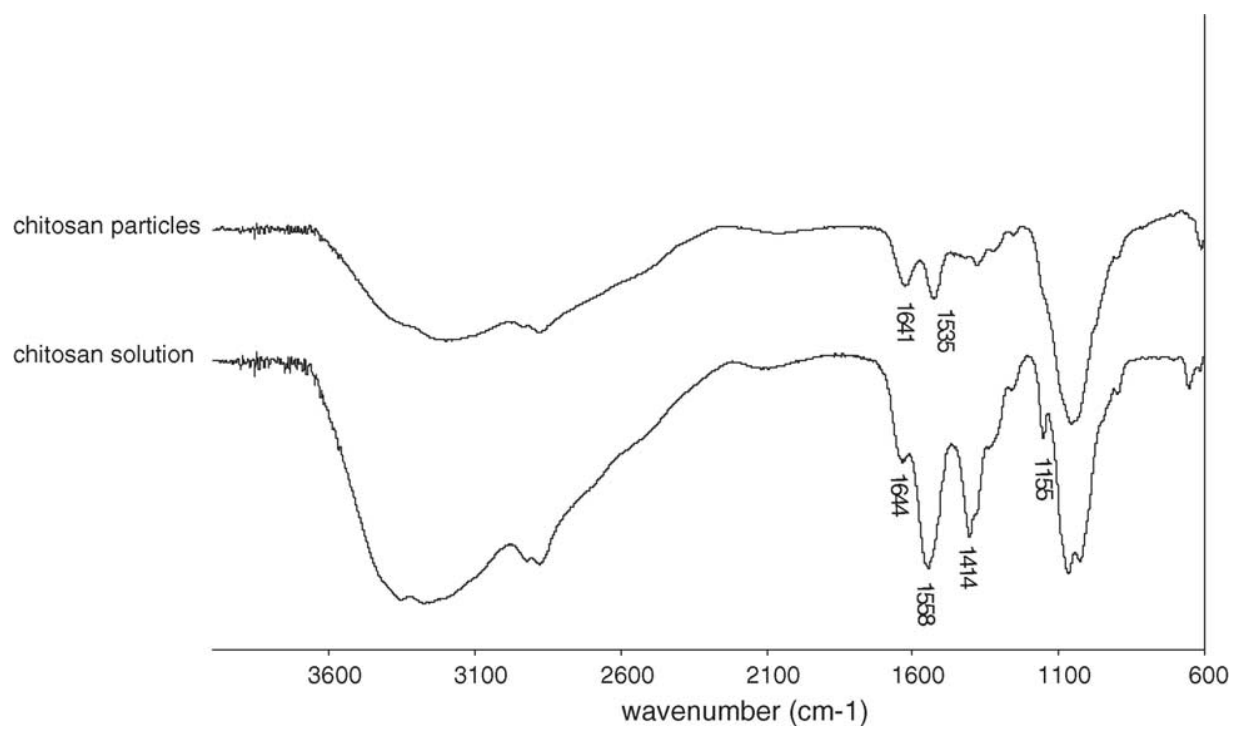

Fig. 6. FTIR spectra of chitosan and unloaded chitosan particles. 


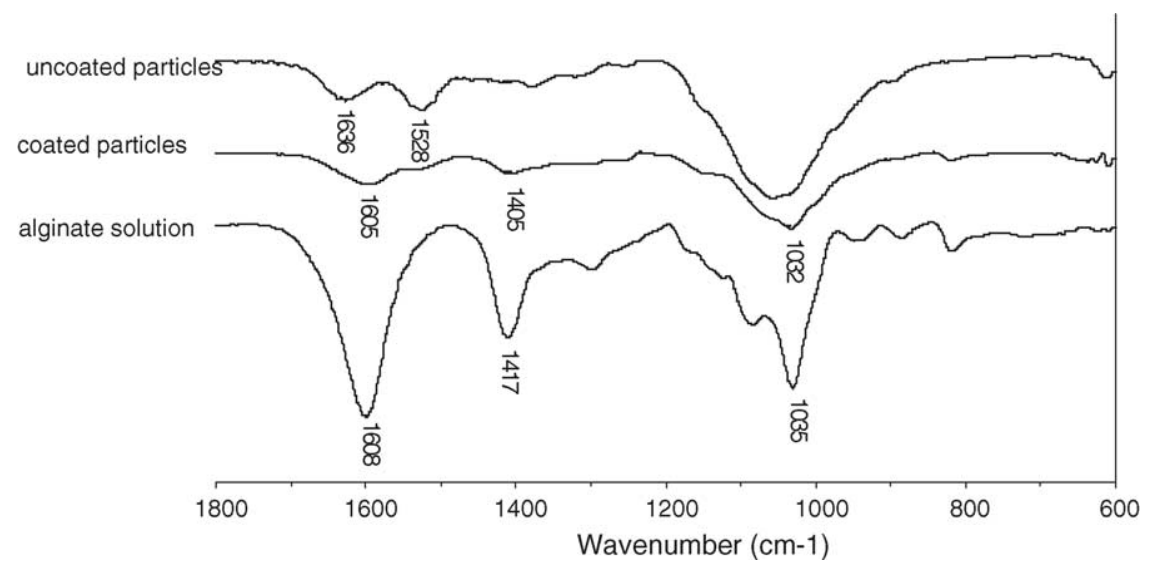

Fig. 7. FTIR spectra of sodium alginate, uncoated chitosan particles and alginate-coated chitosan particles. The FTIR investigation was conducted using the unloaded chitosan particles.

reduced. In addition the peak related with $\mathrm{C}-\mathrm{N}$ stretch $\left(1414 \mathrm{~cm}^{-1}\right)$ has almost disappeared in the chitosan particles. Similar observations are reported for chitosan particles prepared with tripolyphosphate (Xu and $\mathrm{Du}, 2003)$. These observations are in agreement with the fact that the sulphate ions interact with the primary amino groups of the chitosan, resulting in the formation of cross-linked chitosan particles (reticulation process).

Sodium alginate as a carboxylate salt showed a strong asymmetric stretch at $1605 \mathrm{~cm}^{-1}$. The frequency of carbonyl absorption is lowered compared to the value found for the parent carboxylic acid due to a resonance phenomenon. The carboxyl and carboxylate groups are present at wave number of about $1000-1400 \mathrm{~cm}^{-1}$. From the FTIR analysis spectra of the coated particles we were able to distinguish the presence of these three peaks that are different from those of the chitosan uncoated particles. As conclusion of these findings, the results clearly show the existence of alginate coating layer around the chitosan particles.

\subsection{Release studies}

In a preliminary study, we observed that the temperature is an important determinant for the integrity of the chitosan particles placed in different $\mathrm{pH}$ buffers. The particle integrity was monitored by turbidity transmission measurements at $500 \mathrm{~nm}$. In fact at room temperature we were able to obtain stable suspensions of the particles in simulated gastric fluid (SGF) and in simulated intestinal fluid (SIF). In contrast, when the chitosan particles were added to the same solutions (SGF and SIF) at $37^{\circ} \mathrm{C}$, a loss of integrity was observed translated with high values of transmission measurements. Thus, the complete release of the ovalbumin from the chitosan particles in SIF at $37^{\circ} \mathrm{C}$ (Fig. 8) is directly related to the loss of integrity of the particles and less with a simple desorption phenomenon. In contrast, the coating of the particles with alginate increased the stability of the particles (lower values of transmission measurements) in SIF at $37^{\circ} \mathrm{C}$ resulting in a slower release rate of ovalbumin. After $7 \mathrm{~h}$ more than $60 \%$ of ovalbumin was still found in the alginate-coated particles. These release studies also suggest that the formation of an alginate layer increases

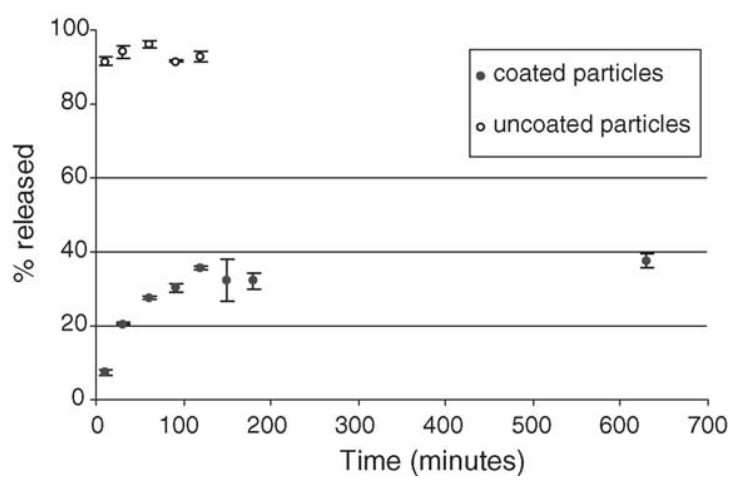

Fig. 8. Release behaviour of ovalbumin from alginate-coated and uncoated chitosan particles in $\mathrm{SIF}$ at $37^{\circ} \mathrm{C}$. Individual points represent the mean averages from three assays. 
Application line

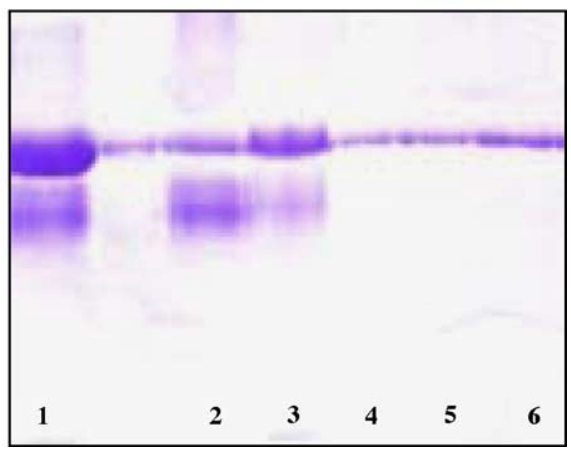

Fig. 9. Electrophoretic analysis on SDS-12\% PAGE with Coomassie brilliant blue staining. Shown are solutions of ovalbumin before conjugation to the nanoparticles (lane 1), ovoalbumin from the supernatant remaining after the conjugation (lane 2), ovoalbumin from the supernatant remaining after the coating with alginate (lane 3), ovoalbumin released from alginate-coated chitosan nanoparticles (lanes 4-6).

the stability of the chitosan particles and reduces the release of the adsorbed protein from these particles.

\subsection{SDS-PAGE}

The main task of this work was the development of an appropriate method for the encapsulation of antigens to an optimised delivery platform. With this method the protein antigen was never exposed to potentially harsh conditions, such as the contact with organic solvents or mechanical agitation or sonication. The SDS-PAGE (Fig. 9) of the released ovalbumin from the particles showed identical bands for the entrapped (lanes 4-6) and native ovalbumin (lane 1) and there were no additional bands to indicate the presence of molecular weight aggregates or fragments greater or less than $45 \mathrm{~K}$ (the molecular weight of ovalbumin). Hence, the data suggest that the structural integrity of ovalbumin was not significantly affected by the entrapment procedure.

\section{Conclusion}

This work describes a new nanosized mucosal vaccine delivery system, consisting of chitosan particles that are prepared by cross-linking with sodium sulphate. The chitosan nanoparticles are loaded under very mild conditions with a model antigen (ovalbumin), which was negatively charged in the used buffer systems. The validity of an easy and economic loading process was shown. In order to increase the stability of the loaded chitosan particles at physiological temperature in SGF and SIF a coating process with sodium alginate was developed. This coating process was optimized in such ways that only small amounts of ovalbumin were desorbed during the coating process and the antigen released from the coated nanoparticles was strongly reduced in comparison to the uncoated chitosan nanoparticles. Hence a nanosized delivery platform is described with improved features of antigen stability in simulated gastrointestinal fluids. In vivo studies are under way to show the efficacy of these systems for mucosal vaccination.

\section{Acknowledgements}

This work was supported by a grant from the Portuguese Ministry of Science and Technology (SFRH/BD/5327/2001). We would like to thank Dr. H. K. Koerten (Faculty of Medicine, Leiden University) for his support with SEM, and to Dr. Alcino Leitao and Dr. João Canotilho (Faculty of Pharmacy, Coimbra University) for the discussions of FTIR spectra and DSC curves, respectively. ISP Alginates (UK).

\section{References}

Alpar, H.O., Eyle, J.E., Williamson, E.D., 1998. Oral and nasal immunization with microencapulated clinically relevant proteins. STP Pharm. Sci. 8, 31-39.

Berthold, A., Cremer, K., Kreuter, J., 1996. Preparation and characterization of chitosan microspheres as drug carrier for prednisolone sodium phosphate as model for anti-inflammatory drugs. J. Control Release 39, 17-25.

Bivas-Benita, M., Laloup, M., Versteyhe, S., Dewit, J., De Braekeleer, J., Jongert, E., Borchard, G., 2003. Generation of Toxoplasma gondii GRA1 protein and DNA vaccine loaded chitosan particles: preparation, characterization, and preliminary in vivo studies. Int. J. Pharm. 266, 17-27.

Bowersock, T.L., HogenEsch, H., Suckow, M., Guimond, P., Martin, S., Borie, D., Torregrosa, S., Park, H., Park, K., 1999. Oral vaccination of animals with antigens encapsulated in alginate microspheres. Vaccine 17, 1804-1811.

Chan, L., Lee, H., Heng, P., 2002. Production of alginate microspheres by internal gelation using an emulsification method. Int. J. Pharm. 242, 259-262. 
Cho, N.H., Seong, S.Y., Chun, K.H., Kim, Y.H., Kwon, I.C., Ahn, B.Y., Jeong, S.Y., 1998. Novel mucosal immunization with polysaccharide-protein conjugates entrapped in alginate microspheres. J. Control Release 53, 215-224.

Coppi, G., Iannuccelli, V., Leo, E., Bernabei, M.T., Cameroni, R., 2002. Protein immobilization in crosslinked alginate microparticles. J. Microencapsul. 19, 37-44.

de Vos, P., Hoogmoed, C.G., Busscher, H.J., 2002. Chemistry and biocompatibility of alginate-PLL capsules for immunoprotection of mammalian cells. J. Biomed. Mater. Res. 60, 252-259.

Eldridge, J.H., Stass, J.K., Meulbroek, J.A., McGhee, J.H., Tice, T.R., Gilley, R.M., 1991. Biodegradable microspheres as a vaccine delivery system. Mol. Immunol. 28, 287-294.

Esquisabel, A., Hernandez, R.M., Igartua, M., Gascon, A.R., Calvo, B., Pedraz, J.L., 2000. Effect of lecithins on BCG-alginate-PLL microcapsule particle size and stability upon storage. J. Microencapsul. 17, 363-372.

Fundueanu, G., Nastruzzi, C., Carpov, A., Desbrieres, J., Rinaudo, M., 1999. Physico-chemical characterization of Ca-alginate microparticles produced with different methods. Biomaterials 20, 1427-1435.

Gonzalez Ferreiro, M., Tillman, L., Hardee, G., Bodmeier, R., 2002. Characterization of alginate/poly-L-lysine particles as antisense oligonucleotide carriers. Int. J. Pharm. 239, 47-59.

Gonzalez-Rodriguez, M.L., Holgado, M.A., Sanchez-Lafuente, C., Rabasco, A.M., Fini, A., 2002. Alginate/chitosan particulate systems for sodium diclofenac release. Int. J. Pharm. 232, 225-234.

Hejazi, R., Amiji, M., 2002. Stomach-specific anti-H. pylori therapy. I. Preparation and characterization of tetracyline-loaded chitosan microspheres. Int. J. Pharm. 235, 87-94.

Jabbal-Gill, I., Lin, W., Jenkins, P., Watts, P., Jimenez, M., Illum, L., Davis, S.S., Wood, J.M., Major, D., Minor, P.D., Li, X., Lavelle, E.C., Coombes, A.G., 1999. Potential of polymeric lamellar substrate particles (PLSP) as adjuvants for vaccines. Vaccine 18 , 238-250.

Jani, P.U., McCarthy, D.E., Florence, A.T., 1992. Nanosphere and microsphere uptake via Peyer's patches: observation of the rate of uptake in the rat after a single oral dose. Int. J. Pharm. 86, 239-246.

Johnson, F.A., Craig, D.Q., Mercer, A.D., 1997. Characterization of the block structure and molecular weight of sodium alginates. J. Pharm. Pharmacol. 49, 639-643.

Kampf, N., Zohar, C., Nussinovitch, A., 2000. Alginate coating of Xenopus laevis embryos. Biotechnol. Prog. 16, 497-505.

Khalid, M.N., Agnely, F., Yagoubi, N., Grossiord, J.L., Couarraze, G., 2002. Water state characterization, swelling behavior, thermal and mechanical properties of chitosan based networks. Eur. J. Pharm. Sci. 15, 425-432.

Kulkarni, A.R., Soppimath, K.S., Aminabhavi, T.M., Rudzinski, W.E., 2001. In-vitro release kinetics of cefadroxil-loaded sodium alginate interpenetrating network beads. Eur. J. Pharm. Biopharm. 51, 127-133.
Machluf, M., Apte, R.N., Regev, O., Cohen, S., 2000. Enhancing the immunogenicity of liposomal hepatitis B surface antigen (HBsAg) by controlling its delivery from polymeric microspheres. J. Pharm. Sci. 89, 1550-1557.

Mi, F.L., Shyu, S.S., Chen, C.T., Schoung, J.Y., 1999. Porous chitosan microsphere for controlling the antigen release of Newcastle disease vaccine: preparation of antigen-adsorbed microsphere and in vitro release. Biomaterials 20, 1603-1612.

Rajaonarivony, M., Vauthier, C., Couarraze, G., Puisieux, F., Couvreur, P., 1993. Development of a new drug carrier made from alginate. J. Pharm. Sci. 82, 912-917.

Ramdas, M., Dileep, K.J., Anitha, Y., 1999. Alginate encapsulted bioadhesive chitosan microspheres for intestinal drug delivery. J. Biomat. Appl. 13, 290-296.

Rebelatto, M.C., Guimond, P., Bowersock, T.L., HogenEsch, H., 2001. Induction of systemic and mucosal immune response in cattle by intranasal administration of pig serum albumin in alginate microparticles. Vet. Immunol. Immunopathol. 83, 93-105.

Roy, K., Mao, H.Q., Huang, S.K., Leong, K.W., 1999. Oral gene delivery with chitosan-DNA nanoparticles generates immunologic protection in a murine model of peanut allergy. Nat. Med. 5, 387-391.

Singh, M., O'Hagan, D., 1998. The preparation and characterization of polymeric antigen delivery systems for oral administration. Adv. Drug. Deliv. Rev. 34, 285-304.

Singh, M., O’Hagan, D., 1999. Advances in vaccine adjuvants. Nat. Biotechnol. 17, 1075-1081.

Takka, S., Acarturk, F., 1999. Calcium alginate microparticles for oral administration. I. Effect of sodium alginate type on drug release and drug entrapment efficiency. J. Microencapsul. 16, 275-290.

Tang, E.S., Huang, M., Lim, L.Y., 2003. Ultrasonication of chitosan and chitosan nanoparticles. Int. J. Pharm. 265, 103-114.

Van der Lubben, I.M., Kersten, G., Fretz, M.M., Beuvery, C., Coos Verhoef, J., Junginger, H.E., 2003. Chitosan microparticles for mucosal vaccination against diphtheria: oral and nasal efficacy studies in mice. Vaccine 21, 1400-1408.

Van der Lubben, I.M., Konings, F.A., Borchard, G., Verhoef, J.C., Junginger, H.E., 2001a. In vivo uptake of chitosan microparticles by murine Peyer's patches: visualization studies using confocal laser scanning microscopy and immunohistochemistry. J. Drug Target. 9, 39-47.

Van der Lubben, I.M., Verhoef, J.C., van Aelst, A.C., Borchard, G., Junginger, H.E., 2001b. Chitosan microparticles for oral vaccination: preparation, characterization and preliminary in vivo uptake studies in murine Peyer's patches. Biomaterials 22, 687-694.

Wu, J.X., Tai, J., Cheung, S.C., Tze, W.J., 1997. Assessment of the protective effect of uncoated alginate microspheres. Transplant. Proc. 29, 2146-2147.

Xu, Y., Du, Y., 2003. Effect of molecular structure of chitosan on protein delivery properties of chitosan nanoparticles. Int. J. Pharm. 250, 215-226. 